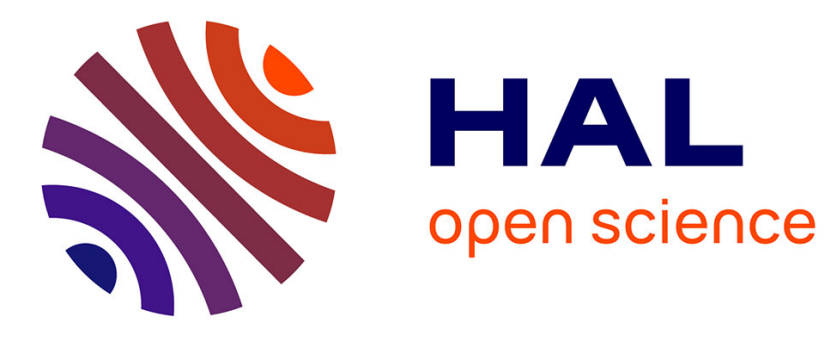

\title{
Enlarged near-field optical imaging
}

Gilles Lerondel, Ahmad Sinno, Luc Chassagne, Sylvain Blaize, Pascal Ruaux, Aurélien Bruyant, Suat Topsu, Pascal Royer, Yasser Alayli

\section{To cite this version:}

Gilles Lerondel, Ahmad Sinno, Luc Chassagne, Sylvain Blaize, Pascal Ruaux, et al.. Enlarged nearfield optical imaging. Journal of Applied Physics, 2009, 106, pp.044913. 10.1063/1.3200953 . hal00830707

\section{HAL Id: hal-00830707 https://hal.science/hal-00830707}

Submitted on 5 Jun 2013

HAL is a multi-disciplinary open access archive for the deposit and dissemination of scientific research documents, whether they are published or not. The documents may come from teaching and research institutions in France or abroad, or from public or private research centers.
L'archive ouverte pluridisciplinaire HAL, est destinée au dépôt et à la diffusion de documents scientifiques de niveau recherche, publiés ou non, émanant des établissements d'enseignement et de recherche français ou étrangers, des laboratoires publics ou privés. 


\title{
Enlarged near-field optical imaging
}

\author{
G. Lérondel, ${ }^{1, a)}$ A. Sinno, ${ }^{2}$ L. Chassagne, ${ }^{2, a)}$ S. Blaize, ${ }^{1}$ P. Ruaux, ${ }^{2}$ A. Bruyant,${ }^{1}$ \\ S. Topçu, ${ }^{2}$ P. Royer, ${ }^{1}$ and Y. Alayli ${ }^{2}$ \\ ${ }^{1}$ Laboratoire de Nanotechnologie et d'Instrumentation Optique, Institut Charles Delaunay, Université de \\ Technologie de Troyes, CNRS FRE 2848, 12 rue Marie Curie, BP 2060, 10010 Troyes Cedex, \\ France \\ ${ }^{2}$ Laboratoire d'Ingénierie des Systèmes de Versailles, EA 4048, Université de Versailles Saint Quentin, 45 \\ avenue des Etats Unis, 78035 Versailles Cedex, France
}

(Received 16 March 2009; accepted 6 July 2009; published online 31 August 2009)

\begin{abstract}
Millimeter scale near-field optical microscopy is here reported. It is demonstrated in the near infrared by observing light propagating in an integrated glass waveguide. Enlarged near-field imaging was made possible thanks to the use of a homemade interferometric translation stage with nanometric scale repeatabilities combined with a commercial atomic force microscope. An integrated optical component was used as a representative example of multiscale components requiring large scale highly resolved optical mapping. By imaging stationary waves on a millimeter long range followed by Fourier analysis, an uncertainty of a few $10^{-4}$ on the waveguide modes effective index was obtained. While improving the optogeometrical parameter retrieval precision, millimeter scans make the technique sensitive to properties such as birefringence. (C) 2009 American Institute of Physics. [DOI: 10.1063/1.3200953]
\end{abstract}

\section{INTRODUCTION}

In conventional scanning near-field microscopy including atomic force microscopy and near-field optical microscopy, the use of piezoelectric stages limits the investigation scale to a few tens of micrometers. This almost prevents the multiscale observation of large nanostructured samples. Among these samples integrated optical waveguiding structures and related scanning near-field optical microscopy (SNOM) appear of special interest. By making possible to observe the light confined in the structure, SNOM is indeed a unique characterization tool for integrated optics and photonic materials. First demonstrated in $1994,{ }^{1}$ today's SNOM phase sensitive measurements allow for quantitative analysis of parameters such as effective index, insertion loss, ${ }^{2}$ group velocity, ${ }^{3}$ and in situ local reflection coefficient ${ }^{4}$ retrieval. While the potential of the technique is now demonstrated, the measurement accuracy remains directly limited by the scanning area. In order to improve the accuracy and make the observation of the entire structure possible (chip scale), millimeter size near-field optical imaging is required. While this has been demonstrated by stitching micrometer scale images together, ${ }^{5}$ the stitching approach is, however, time consuming and the accuracy relies on topographical patterns (defects). Recently, we demonstrated by using a twofold equipment combining a commercial atomic force microscope (AFM) and a homemade piezomicrointerferometric stage that millimeter range AFM scanning is possible. ${ }^{6}$ While large scale AFMs are under development in the major worldwide metrological institutes, ${ }^{7-12}$ large scale near-field optical imaging has not yet been reported. In this paper, we show that millimeter scale optical near-field imaging is also possible. As an example, the light propagation in a glass integrated

\footnotetext{
${ }^{a)}$ Authors to whom correspondence should be addressed. Electronic addresses: gilles.lerondel@utt.fr and luc.chassagne@uvsq.fr.
}

waveguide has been investigated. The numerous advantages of multiscale highly resolved optical imaging will be discussed based on experimental results.

\section{EXPERIMENTAL DETAILS}

Figure 1 shows a schematic view of the enlarged nearfield optical microscope. The system consists in a homemade interferometric stage combined with a commercial AFM (Park Scientific Instrument). The AFM tip working in tapping mode provides the near-field information while a heterodyne laser interferometry allows to measure and to control the sample position with nanometric scale repeatabilities. Details of the homemade interferometric stage and its integration under the AFM can be found in Ref. 6. The optical laser source used in the experiment is a tunable external cavity laser (Tunics) allowing an absolute wavelength uncertainty of $\pm 5 \mathrm{pm}$. Observations were done at $1.55000 \mu \mathrm{m}$.

In order to test the enlarged scanning optical microscope, we characterized light propagation along a single-mode ion exchange waveguide. The sample was fabricated using a two-step $\mathrm{Ag}+/ \mathrm{Na}+$ ion exchange process in a silicate glass substrate and buried $3 \mu \mathrm{m}$ beneath the glass top surface by means of a thermal rediffusion process. The guide was formed through a $1.7 \mu \mathrm{m}$ diffusion aperture width. The diffusion process produces a graded index profile which cannot be accurately measured for this type of confined waveguide. The index variation is empirically estimated to be of the order of $10^{-2}$ above the substrate refractive index value of 1.51. Further details on such sample and the fabrication process are given in Ref. 13.

The sketch in the insert in Fig. 1 shows the typical dimensions involved in the experiment. The sample under investigation is a $2 \mathrm{~cm}$ long straight waveguide while its width given by the diffusion aperture is only $1.7 \mu \mathrm{m}$. At last, the near-field information is provided via the AFM tip oscillating 


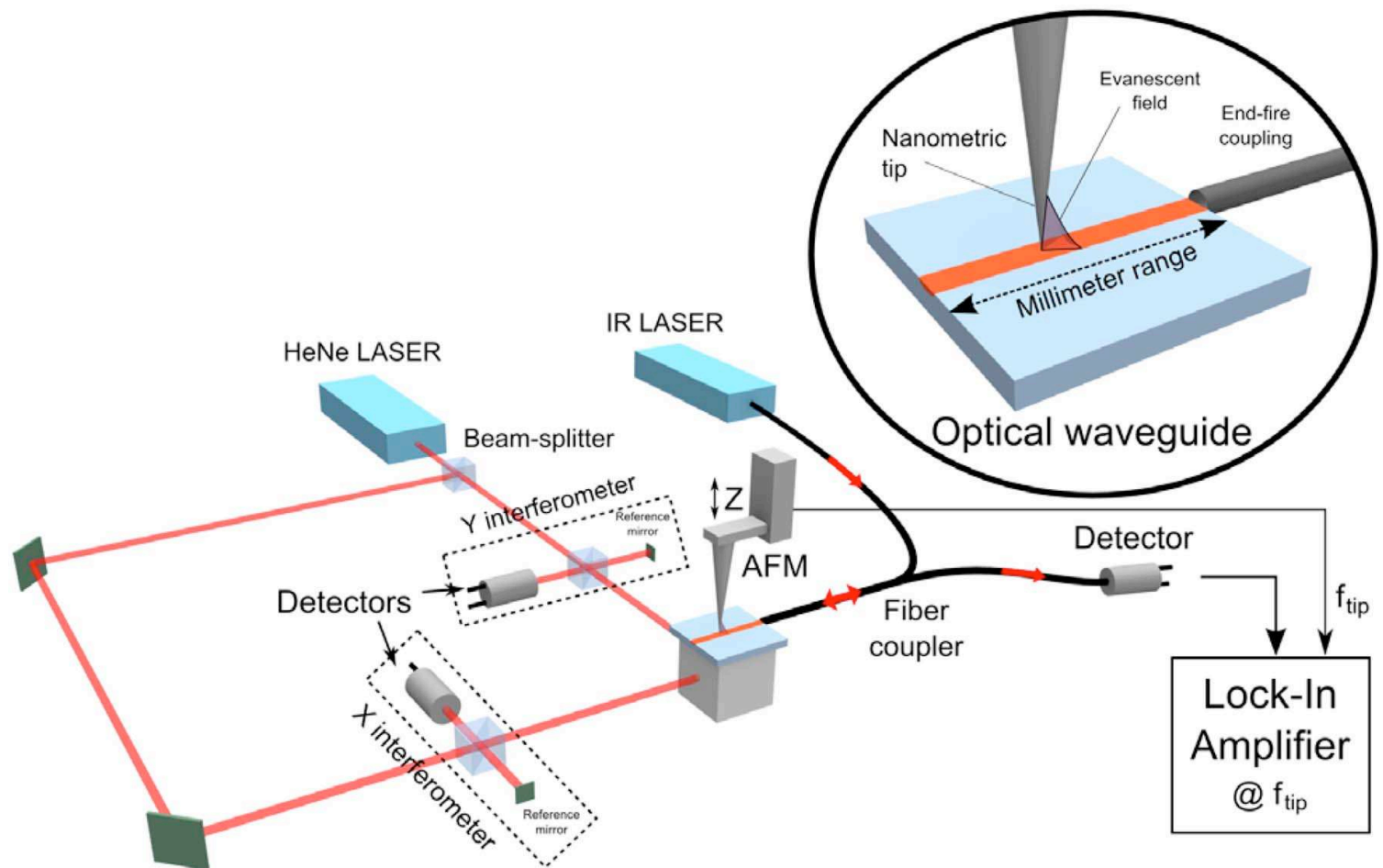

FIG. 1. (Color online) Schematic view of the long range near-field scanning optical microscope. The two dimensional (2D) millimeter scale displacement is achieved thanks to a two linear motors actuated $X$ and $Y$ stages whose positions are tracked in both directions using optical interferometers. A 2D piezoelectric actuator allows for displacement deviations to be corrected. The sample under investigation is a single-mode channel integrated waveguide. The optical near-field information (cf. insert) is provided using an AFM tip working in tapping mode (scattering type of SNOM).

with an amplitude of $100 \mathrm{~nm}$. The waveguide is metallized at one extremity in order to enhance the reflexion at the exit of the waveguide and thus induce stationary waves within the guiding structure.

To simplify the optical detection scheme and following the idea of the recently proposed integrated detection transmission SNOM ${ }^{14}$ the AFM-tip modulated signal is here detected in reflection using the counterpropagating mode as the modulated signal vector. As shown in Fig. 1, this is made possible thanks to a $Y$ splitter used for both injection and collection.

As already discussed in Ref. 6 three possible scanning modes can be used for enlarged scanning near-field microscopy. The $X$ and $Y$ directions will thereafter refer to the fast and slow scanning directions, respectively. The so-called line by line mode was used. Scans in the $X$ direction are performed with the linear motors without piezoelectric compensation along the displacement axis. The piezoelectric stage only compensates the straightness along the $Y$ axis. Thanks to a fast acquisition board, the AFM acquisition is synchronized with the linear motor motion. This mode is not optimized for position control but for acquisition time.

Two scanning near-field optical images, hereafter referred to as A and B, are presented. The image A is composed of $27100 \mu \mathrm{m}$ long scans spaced by $300 \mathrm{~nm}$. A scanning speed of $5 \mu \mathrm{m} / \mathrm{s}$ and an acquisition interval of $60 \mathrm{~nm}$ were used. The image B is composed of $2001.1 \mathrm{~mm}$ long scans spaced by $150 \mathrm{~nm}$. A scanning speed of $30 \mu \mathrm{m} / \mathrm{s}$ and an acquisition interval of $100 \mathrm{~nm}$ were used. The total fields of view for images $\mathrm{A}$ and $\mathrm{B}$ are thus $100 \times 8$ and 1.1 $\times 30 \mu \mathrm{m}^{2}$, respectively. In the case of SNOM imaging the optical signal to noise ratio has also to be considered. Images $\mathrm{A}$ and $\mathrm{B}$ have been obtained with integration times on the lock-in amplifier of 5 and $2 \mathrm{~ms}$, respectively.

\section{RESULTS AND DISCUSSION}

Figure 2(a) shows a typical $100 \mu \mathrm{m}$ long optical image (image A). A clear intensity modulation can be observed along the fast scan direction. Figure 2(b) shows the averaged fast Fourier transform (FFT) along that direction. From this type of Fourier transform, modal analysis can be performed and important optical characteristics of the waveguide can be retrieved such as the number and the effective index of the optical modes supported by the waveguide. ${ }^{15}$ The intensity modulation clearly observable on the inset corresponding to

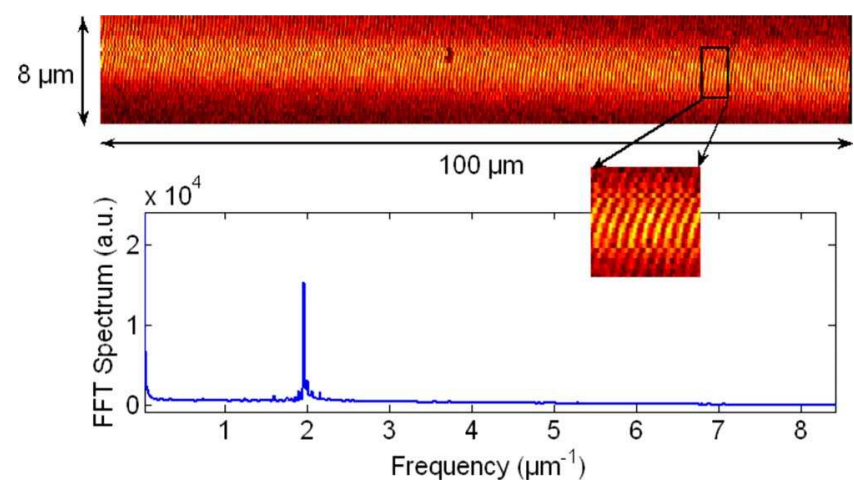

FIG. 2. (Color online) $100 \mu \mathrm{m}$ scale test sample image. (a) $100 \mu \mathrm{m}$ $\times 8 \mu \mathrm{m}$ near-field optical image and (b) corresponding line by line averaged FFT analysis. FFT analysis allows for a precise measurement of the interference pattern (cf. zoom) periodicity. 


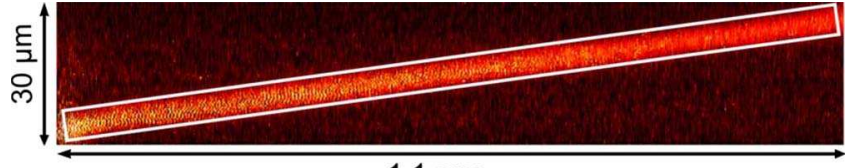

$1.1 \mathrm{~mm}$

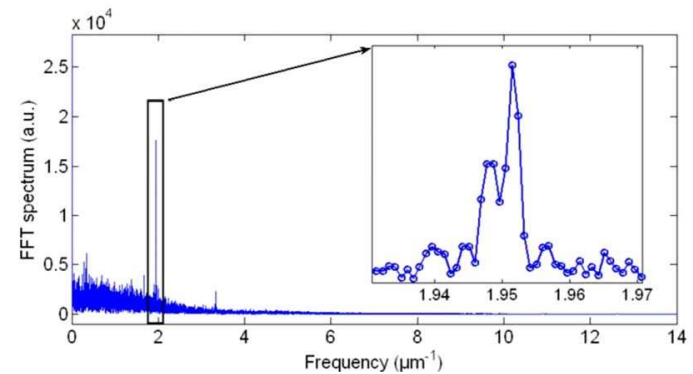

FIG. 3. (Color online) Millimeter scale image: (a) $1100 \mu \mathrm{m} \times 30 \mu \mathrm{m}$ nearfield optical image and (b) corresponding line by line averaged FFT analysis

a zoomed area evidences the interference of forward and backward propagating modes. The single peak on the FFT confirms that at $1.55 \mu \mathrm{m}$ the waveguide only supports a single mode. From the FFT peak position, the intensity modulation periodicity $p$ can be measured, and from there, the propagation wavelength of the guided light $\lambda_{G}$ $=1025 \pm 6 \mathrm{~nm}$. The uncertainty on the peak position measurement is deduced from the spectral width [full width at half maximum (FWHM)] of the FFT. Taking into account the optical wavelength $\left(\lambda_{0}=1.55000 \mu \mathrm{m}\right)$ the effective index of the mode and uncertainty are straightforwardly obtained using the following equations:

$$
\begin{aligned}
& n_{\text {eff }}=\frac{\lambda_{0}}{\lambda_{G}}=\frac{\lambda_{0}}{2 p}, \\
& \Delta n_{\text {eff-FWHM }}=\Delta \lambda_{G} \frac{\lambda_{0}}{\lambda_{G}^{2}},
\end{aligned}
$$

where $\Delta \lambda_{\mathrm{G}}$ is the FWHM measured on the curve. One finds here 1.512 \pm 0.009 in agreement with previous SNOM based modal analysis. ${ }^{15}$

Figure 3(a) shows a $1.1 \mathrm{~mm}$ long near-field image (image $\mathrm{B}$ ). To the best of our knowledge this is the largest nearfield optical image ever realized. Actually, we already reported a $2 \mathrm{~mm}$ long mapping of a glass integrated $Y$ junction but this was achieved by stitching 25 images of 50 $\times 20 \mu \mathrm{m}^{2}$ each. ${ }^{5}$ Besides being time consuming, the stitching approach is only possible if topographical details are available to ensure accurate stitching of the images together. Figure 3(b) shows the average Fourier transform of the adjacent scans. As for the previous image, similar information on the optogeometrical parameters can be retrieved but, due to a longer scan length, with a better resolution. Surprisingly, one finds here two peaks instead of one. As for the image 2(b), from the peak positions and FWHM the guided wavelengths can be retrieved; for the left peak, $\lambda_{G 1}$ $=1026.5 \pm 0.8 \mathrm{~nm}$ and $n_{\mathrm{eff} 1}=1.510 \pm 0.001$, while for the right peak $\lambda_{G 2}=1024.6 \pm 0.6 \mathrm{~nm}$ and $n_{\mathrm{eff} 2}=1.5126 \pm 0.0009$. Before discussing the origin of these two peaks let us discuss the precision of the measurement.
With an uncertainty on the refractive index of less than $10^{-3}$, SNOM based modal analysis appears definitively as an alternative qualitative characterization technique for integrated waveguiding samples.

Based on Fourier transform, the resolution of the modal analysis using SNOM is directly related to the scanning length while the bandwidth is limited by the sampling frequency. Therefore, to improve the accuracy while keeping the bandwidth the same, there is an obvious interest of increasing the scanning area while preserving the scan resolution.

If we assume that the accuracy on the FFT peak position $(1 / p)$ is $1 / L$ (FFT sampling interval) with $L$ the scan length, using Eq. (1), one can easily show that the effective index resolution $r$ is directly related to the scan length by the following relation:

$$
r=\frac{\lambda_{0}}{2 L},
$$

with $\lambda_{0}$ the wavelength in air $(1.55000 \mu \mathrm{m})$. The contribution of the laser source spectral width is here neglected. Dealing with a discrete measurement, we verify here that the Nyquist-Shannon sampling criterion is satisfied. The sampling intervals for images A and B were, respectively, 60 and $150 \mathrm{~nm}$ which have to be compared to the periodicity of the stationary wave of about $500 \mathrm{~nm}$. At last, due to the sample symmetry, the measured signal to noise ratio can also be increased by averaging the FFT of the different scanned lines. It is important to note that averaging will only be efficient on the waveguiding area.

Using Eq. (3), the resolution of each scan length can be estimated. One finds for image A, corresponding to the $100 \mu \mathrm{m}$ scan length, $r=0.008$ which has to be compared with the experimental deviation deduced from the FFT linewidth, 0.009. For image B instead, corresponding to the 1.1 $\mathrm{mm}$ long scan length, the expected $r$ is equal to 0.0007 which in this case has to be compared to 0.0009 . In both cases, the experimental uncertainty was found to be almost equal to the expected theoretical resolution. As already discussed in Ref. 6 , various issues have to be addressed when dealing with a millimeter scale near-field microscopy. These include temperature fluctuations that induced drifts, sample flatness, and sample alignment along the scanning direction. The remarkable agreement obtained between the experimental result and the theoretical calculation shows that none of these effects has strongly affected the measurement.

A last point to be addressed is the double peak structure revealed by the FFT of the millimeter scan [cf. Fig. 3(b)]. From the two peak positions, the effective index variation can be estimated and was found to be $0.0027 \pm 0.0019$. This difference is attributed to the birefringence of the waveguide. Following the ion exchange process a thermal treatment is used to burry the waveguide, but due to a remaining refractive index profile asymmetry, a birefringence in the order of $10^{-3}$ is still expected in such waveguide. ${ }^{16}$ 


\section{CONCLUSIONS}

A millimeter scale near-field optical mapping has been demonstrated by observing the light propagation within a centimeter long channel optical waveguide. Compared to conventional near-field microscopy, the enlarged near-field optical microscopy combined with a Fourier analysis allows for a more accurate retrieval of the optogeometrical parameters $\left(10^{-4}\right)$. Such an accuracy enlarges the scope of nearfield optical microscopy by making the technique also sensitive to extremely low birefringence. While millimeter scale near-field imaging has been demonstrated using a waveguiding sample, the technique is not limited to this type of samples neither to the integrated detection scheme. It is fully compatible with any type of SNOM mode including aperture types and scattering types. Besides chip scale characterization, the reported technique has the potential to broaden the field of near-field microscopy by making the multiscale lightmatter interaction probing possible and introducing near-field optical metrology.

\section{ACKNOWLEDGMENTS}

The authors are grateful to Loic Le Cunf for his help preparing Fig. 1. This work was supported by the French ministry for research through the "nano2m3" integrated project, the "Région Champagne-Ardenne," and the European Regional Development Fund (ERDF).

${ }^{1}$ A. G. Choo and H. E. Jackson, Appl. Phys. Lett. 65, 947 (1994).

${ }^{2}$ I. Stefanon, S. Blaize, A. Bruyant, S. Aubert, G. Lerondel, R. Bachelot, and P. Royer, Opt. Express 13, 5553 (2005).

${ }^{3}$ M. L. M. Balistreri, H. Gersen, J. P. Korterik, L. Kuipers, and N. F. van Hulst, Science 294, 1080 (2001).

${ }^{4}$ A. Bruyant, G. Lérondel, S. Blaize, I. Stefanon, S. Aubert, R. Bachelot, and P. Royer, Phys. Rev. B 74, 075414 (2006).

${ }^{5}$ R. Bachelot, G. Lérondel, S. Blaize, S. Aubert, A. Bruyant, and P. Royer, Microsc. Res. Tech. 64, 441 (2004).

${ }^{6}$ A. Sinno, P. Ruaux, L. Chassagne, S. Topçu, Y. Alayli, G. Lerondel, S. Blaize, A. Bruyant, and P. Royer, Rev. Sci. Instrum. 78, 095107 (2007).

${ }^{7}$ J. Haycocks and K. Jackson, Precis. Eng. 29, 168 (2005).

${ }^{8}$ R. Leach, J. Haycocks, K. Jackson, A. Lewis, S. Oldfield, and A. Yaccot, Nanotechnology 12, R1 (2001).

${ }^{9}$ G. Wilkening and L. Koenders, Nanoscale Calibration Standards and Methods (Wiley-VCH, New York, 2005).

${ }^{10}$ F. Meli and R. Thalmann, Meas. Sci. Technol. 9, 1087 (1998).

${ }^{11}$ M. Holmes, R. Hocken, and D. Trumper, Precis. Eng. 24, 191 (2000).

${ }^{12}$ J. A. Kramar, Meas. Sci. Technol. 16, 2121 (2005).

${ }^{13}$ J.-E. Broquin, Integrated Optics Devices Proceedings of SPIE, edited by V. Giancarlo and C. Righini, 2001 (unpublished), Vol. 4277, pp. 105-115.

${ }^{14}$ J. T. Robinson, S. F. Preble, and M. Lipson, Opt. Express 14, 10588 (2006).

${ }^{15}$ S. Blaize, S. Aubert, A. Bruyant, R. Bachelot, G. Lerondel, P. Royer, J.-E. Broquin, and V. Minier, J. Microsc. 209, 155 (2003).

${ }^{16}$ R. V. Ramaswamy and R. Srivastava, J. Lightwave Technol. 6, 984 (1988). 